\title{
Negative congruence effects in letter and pseudo-letter recognition: the role of similarity and response conflict
}

Received: 15 July 2004 / Revised: 24 September 2004/ Accepted: 27 September 2004/Published online: 3 November 2004

(C) Marta Olivetti Belardinelli and Springer-Verlag 2004

\begin{abstract}
Letters and pseudo-letters were presented in three experiments using a sequential same-different task. While first items were always presented in isolation, the second item was either presented in isolation or surrounded by geometrical non-target shapes that could be congruent or incongruent to the target. In two experiments, a physical sameness criterion was used. In Experiment 1, in one condition, different pairs were always distinct in shape, in another they were similar in shape. Negative congruence effects were obtained for different pairs that are similar. In Experiment 2, this effect is replicated within participants. In this experiment, similar and dissimilar stimuli were mixed. The results were explained in terms of the difficulty of responding different to stimuli that are similar in shape: when the second item is surrounded by a congruent shape, the similarity is emphasized, making this response even more difficult. In Experiment 3, the same stimuli were presented using a categorical sameness criterion. This reduces the role of physical similarity and thus eliminates the response conflict. As a result, negative congruence effects were no longer observed. Taken together, the three experiments identify another source of negative congruence effects besides the ones recently reported in the literature.
\end{abstract}

Keywords Congruence effects - Letter recognition · Similarity $\cdot$ Response conflict $\cdot$ Flanker $\cdot$ Same-different comparison

\footnotetext{
T. Lachmann ( $\square)$

Department of Psychology, University of Leipzig,

Seeburgstraße 14/20, 04103 Leipzig, Germany

E-mail: lachmann@rz.uni-leipzig.de

T. Lachmann · C. van Leeuwen

Laboratory for Perceptual Dynamics,

Brain Science Institute, RIKEN, Wako-Shi, Japan

C. van Leeuwen

University of Sunderland, Sunderland, UK
}

\section{Introduction}

The perception of graphics is likely to be influenced by a surrounding frame (as in Fig. 1). In peripheral vision, when the distracter information is sufficiently close, the frame may disturb perception as a result of insufficient resolution due to receptor spacing or retino-cortical transmission pooling. Disturbances, however, still occur in peripheral vision when the separation is increased (Bouma 1970; Hagenaar and van der Heijden 1986; Miller 1991; Toet and Levi 1992). They also persist with increased separation in parafoveal and foveal vision, although more restricted than for peripheral vision (Leat et al. 1999). Because the spatial tuning functions of these effects are similar to those of masking (Chung et al. 2001), they are generally attributed to early visual processing and have become known as lateral masking.

Lateral masking has traditionally been explained through interaction of flanking items at the level of local features (Estes 1972). As a consequence, the lateral masking effect was larger when the flankers were similar to the targets (Estes 1982). In the repeated-letter inferiority effect (Bjork and Murray 1977; Egeth and Santee 1981), for instance, it is more difficult to report the leftmost "A" in "AA" than in "AE" conditions. When it is more difficult to report a target if nearby non-targets are similar in shape than when they are different, we speak of a negative congruence effect. Local feature interaction, therefore, may be a possible cause of this effect.

Pomerantz et al. (1988) showed that neighbouring non-targets can sometimes result in positive congruence effects as well. Flanker features may become integrated with the target to form a global whole that is easy to detect. For instance, when the leftmost parenthesis is the target in the configuration "))", it is easy to respond. The integration may take place automatically, prior to target selection or later. These effects are characteristic of simple stimuli, because when the task is easy (Lavie 1995), attention recruited for feature integration 

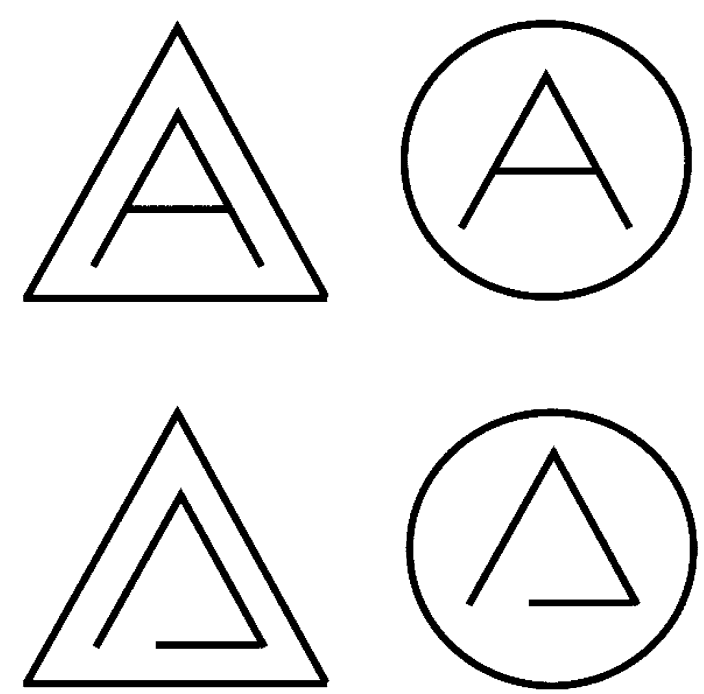

Fig. 1 Letters (upper half) versus pseudo-letters (lower half) in congruent (left) versus incongruent (right) surroundings

(Treisman and Gelade 1980) spills over to irrelevant stimulus attributes. Congruence effects may, of course, also result when incongruent flankers compete. When a flanker calls for a response opposite to that of the target, response competition will result (Eriksen and Eriksen 1974). In general, this will yield a net advantage for congruent stimuli. In sum, the effects of irrelevant surrounding information are commonly explained as a combination of early interference between features, early target selection, and late effects such as response competition (Rueckl et al. 1991; Sanders and Lamers 2002).

The results described so far support the notion of perception as a linear progression from early processes such as feature interaction, subsequent feature integration, and late processes of interference at the response level. Effects of high-level categories operate, in this view, at the response level.

Not all negative congruence effects fit the linear framework. Some cannot be completely understood without considering the possibility of high-level influence of categorical information on early processes such as feature interaction and integration (Bavelier et al. 2000; Briand 1994; van Leeuwen and Bakker 1995; van Leeuwen and Lachmann 2004). In the most recent study, letters and shapes were presented in a choice-response task, surrounded by irrelevant flanker information. The stimuli were foveated and presented in a spatial resolution that excluded lateral masking. A positive congruence effect was obtained for shapes, but a negative congruence effect for letters.

These contrasting results were explained in terms of different feature integration strategies for letters and non-letter shapes (Freeman et al. 2003). Positive congruence effects for non-letter shapes indicate that these are preferably encoded holistically (Pomerantz et al. 1988). For letters, analytic visual encoding is preferred (Nazir et al. 1998). This reduces interference from spurious flanker information with the abstract letter code (Posner 1978). The results, therefore, demonstrate how high-level categorical distinctions can influence early feature integration leading to opposite effects.

An analytic preference would normally lead to absence of (positive or negative) congruence effects. But as perceptual encoding strategies are not mutually exclusive, we may expect that the holistic shape still interferes with the analytic encoding strategy. This is more strongly the case if the surrounding shape is similar to the target. The negative congruence effect in van Leeuwen and Lachmann (2004), therefore, is best understood as rivalry between alternative perceptual strategies.

Because the effect in van Leeuwen and Lachmann (2004) is of strategic nature it is very sensitive to taskspecific maniputations involving the similarity. In particular, similarity between items in the response category was of great importance for the occurrence of negative congruence effects. The negative congruence effect for letters was obtained when items in different categories were visually similar to each other. This result underlines the strategic character of feature integration. Because items in different response categories were similar, their visual shape was not a reliable response criterion. This means that the distinction had to be made, based on their distinctive semantic content: letters versus nonletters. Thus, the distinction was made by invoking the letter strategy for letters, in contrast with the holistic shape strategy for shapes.

When items within the same categories were visually similar to each other, negative congruence effects were not obtained. Instead, there were positive congruence effects for both letters and non-letter shapes. Visual similarity within response categories implies that the items were classified by their visual shape, allowing a holistic strategy for both. Thus, the extent to which visual similarity occurs between or within response categories is crucial for the choice of perceptual strategy.

The aim of the present study is to extend the scope of our investigation to include the role of working memory. Target stimuli in congruent or incongruent surroundings were preceded by another stimulus. First and second stimuli were letters and pseudo-letter shapes. To assure the relevance of the first stimulus, the task was changed to a same-different task (Nickerson 1969). In the samedifferent task, one item is compared to a single probe that is either presented simultaneously (Keren et al. 1977) or successively (Sanders and Lamers 2002), in order to decide whether it is the same or different. Ours was a sequential task, with a long inter-stimulus interval (ISI). The first stimulus was presented sufficiently long to establish its encoding, but masked in order to eliminate visual persistence, so that a working-memory representation remained.

Most congruence effects obtained in choice responses generalize to the same-different task (Sanders and 
Lamers 2002). We expected, however, that the negative congruence effects of van Leeuwen and Lachmann (2004) will not generalize straightforwardly to the sequential same-different task. The reason is that the same-different task imposes an additional relationship of form on the stimuli, the relation between the first and second stimulus. This may be illustrated by an effect that is characteristic of the same-difference task, the fast-same effect (Krueger 1973; Proctor 1981). When the first and the second item are identical, the response is much faster than when they are different. This effect occurs, even when the task is a categorical same-different task, in which participants respond with same also to different members of the same category.

In the physical sameness tasks, where the same response is reserved for identical items there is, as a result of this relationship, an important negative effect of similarity of the first and second item. When two items that are similar but non-identical are compared, their similarity requires a same response. At the same time, their non-identity calls for a different response. We observed a response conflict under these conditions in the same-different task (Lachmann and van Leeuwen 2004a, c). The role of similarity in the same-different task is potentially equally crucial. However, it operates differently than in the choice-response task. Whereas in the choice-response task it determines perceptual strategy, in the same-different task it induces response conflict. This means that we may expect to observe negative congruence effects under different conditions than in the choiceresponse task.

\section{Experiment 1}

Method

\section{Participants}

There were 40 student volunteers ( 31 female) from the University of Leipzig between 20 and 32 years old, who were either paid or received course credits for their participation. All participants had normal or corrected to normal vision and hearing.

\section{Stimuli}

A subset of the targets from van Leeuwen and Lachmann (2004) was used: the three capital letters $A, L$, and $C$ and three corresponding pseudo-letters. The targets are shown in the leftmost columns of Fig. 2. Targets were presented in isolation or surrounded by one of three non-target geometrical shapes. These were either a triangle, a rectangle, or a circle. Targets and non-targets were combined in such a manner that the surrounding shapes could be either congruent or incongruent with respect to the shape of the target. In total, there were six sets of stimuli: isolated letters, letters within congruent surrounding shapes, letters within incongruent sur-
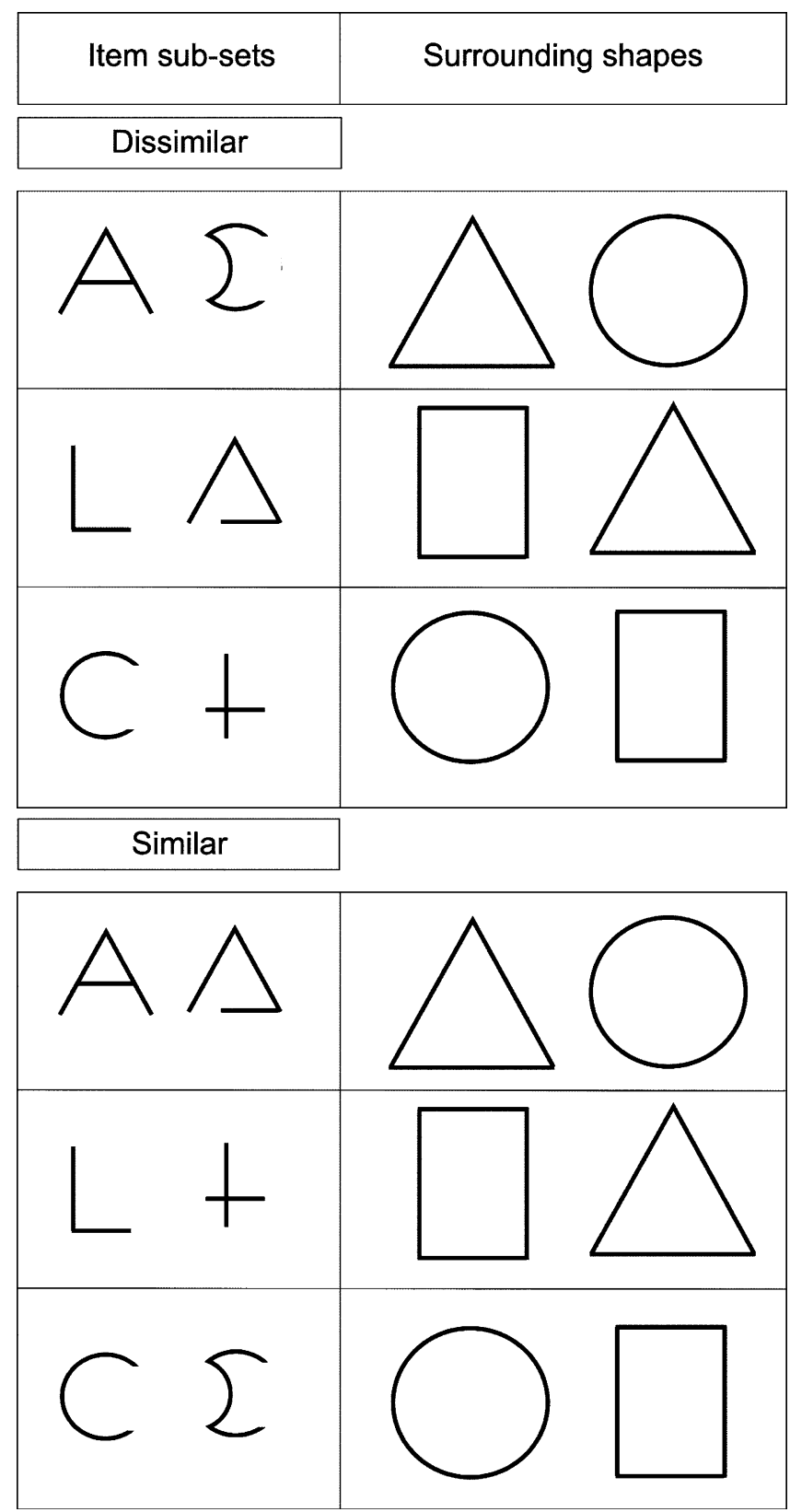

Fig. 2 Targets (letters: leftmost column and pseudo-letters: second column from left) and their surroundings (rightmost two columns). First three rows describe the subsets used to generate the stimuli of the Dissimilar group, last three rows describe the subsets used to generate the stimuli of the Similar group. See text for explanation

rounding shapes, isolated pseudo-letters, pseudo-letters within congruent surrounding shapes, and pseudo-letters within incongruent surrounding shapes (see Fig. 2). Each set contained three stimuli, resulting in a total of 18 unique displays.

Stimuli were scaled to an imaginary $40 \times 40 \mathrm{~mm}$ matrix and the surrounding shapes within an imaginary $80 \times 80 \mathrm{~mm}$ matrix. All stimuli were presented in white $\left(28 \mathrm{~cd} / \mathrm{m}^{2}\right)$ on a CRT computer monitor screen set to black $\left(0.46 \mathrm{~cd} / \mathrm{m}^{2}\right)$. They were presented at about $60-\mathrm{cm}$ length distance, resulting in a visual angle of about $3.5^{\circ}$ 
without and about $7.5^{\circ}$ with surrounding. Participants were seated in a chair, without fixation of their heads. The experiment took place in a dimly lit room without windows.

\section{Procedure}

Participants were instructed to classify as fast and as accurately as possible whether two successively presented items were either same or different and to ignore the surrounding shape, whenever one occurred, as irrelevant. The response was given by pressing one of two response keys on the computer keyboard. Responsekey allocation was counterbalanced across participants. Prior to the experiment, participants performed 30 practice trials using the same procedure as in the experiment, which was as follows. First, a fixation cross $(10 \times 10 \mathrm{~mm})$ occurred for $50 \mathrm{~ms}$ at the left half of the screen, at the position that marked where the target stimuli would follow. After that the screen remained clear for $500 \mathrm{~ms}$. Then the first target stimulus was presented for $800 \mathrm{~ms}$, followed by a mask for $3 \mathrm{~ms}$ (as a theoretical value that cannot be reached by the system). After an interval of $647 \mathrm{~ms}$ during which the screen remained empty, a second fixation cross $(10 \times 10 \mathrm{~mm})$ occurred for $50 \mathrm{~ms}$ in the right half of the screen, marking the place where $500 \mathrm{~ms}$ later the second stimulus was shown until the response of the participant. After the reaction another mask appeared, which was shown for $3 \mathrm{~ms}$, subsequently the screen remained clear for 2,000 ms until the next trial started. Feedback on speed and accuracy was given in the practice trials. No feedback was given in experimental trials.

The first stimulus was always presented without a surrounding, while the second stimulus could occur isolated, within a congruent surrounding, or within an incongruent surrounding. Material classes of the first and second stimulus of a pair were varied independently. That is, a letter could be followed by a letter or a pseudo-letter, and vice versa, a pseudo-letter could be followed by pseudo-letter or a letter. This resulted in four different pairs: letter/letter, letter/pseudo-letter, pseudo-letter/letter, and pseudo-letter/pseudo-letter. Consequently, same responses always contained either letters only or pseudo-letters only, whereas the materials were mixed for different responses.

There were two groups of participants. The set of trials presented was varied between participants. The same pairs were identical for both groups; the different pairs varied between the groups. For the Dissimilar group $(n=19)$, the different stimuli were distinctive in form (e.g. the letter $A$ followed the pseudo-letter $C$ ). For the Similar group $(n=21)$, the different stimuli were similar to each other in form (e.g. the letter $A$ followed the pseudo-letter $A$ ). The Dissimilar group received all possible combinations of pairs from within the three dissimilar target subsets in Fig. 2. The Similar group received all possible combinations of pairs from within the three similar target subsets in Fig. 2. All other conditions were the same for both groups.

For both the Dissimilar and the Similar group, there were 3 (subsets) $\times 4$ (combinations per subset) $\times 3$ (isolated vs. congruent vs. incongruent) $=36$ unique stimulus pairs. Half of these were same pairs and the other half were different. During one block the 36 pairs of stimuli were presented in fully random order. There were six blocks with a short break in-between, resulting in a total of 216 responses for each participant. The whole experimental session, consisting of the introduction, the training, and the test session including breaks, lasted about $35 \mathrm{~min}$.

\section{Results}

A total of 8,640 responses were collected. Responses were removed as outliers from the data set when the reaction time (RT) was shorter than $140 \mathrm{~ms}$ or longer than 3,000 ms. Of the remaining RTs, those that exceeded the criterion of $\mathrm{M}+3.5 \mathrm{SD}$ were removed as well $(\mathrm{M}=$ mean; $\mathrm{SD}=$ standard deviation $)$. According to this procedure, $0.5 \%$ of the cases were excluded from further analyses, which were equally distributed over the experimental conditions and individuals. Only correct responses were used for RT analyses. The overall mean RT was $503 \mathrm{~ms}(\mathrm{SD}=180 \mathrm{~ms})$ and the mean error rate was $5.1 \%$. Mean RT and error rates were not correlated. For this reason, analyses of variance (ANOVA) were performed on RT data only. The data for all conditions are displayed in Table 1.

A first ANOVA was performed using the two withinsubject factors Surrounding (isolated, surrounded), Pair (letter/letter, letter/pseudo-letter, pseudo-letter/letter, and pseudo-letter/pseudo-letter) and the between-subjects factor Similarity (similar, dissimilar). Significant main effects were found for Surrounding, $F(1$, $38)=82.53, p<0.001$, and for Pair, $F(3,114)=39.27$, $p<0.001$. No main effect of Similarity was obtained in
Table 1 Mean reaction times (ms) for same-different pairs, in isolated, congruent and incongruent conditions, displayed separately for the Dissimilar and Similar groups in Experiment 1

\begin{tabular}{llllllll}
\hline & \multicolumn{5}{l}{ Dissimilar } & & \multicolumn{2}{l}{ Similar } \\
\cline { 2 - 3 } & Isolated & Congruent & Incongruent & & Isolated & Congruent & Incongruent \\
\hline Letter/letter & 444 & 463 & 466 & & 426 & 462 & 468 \\
Letter/Pseudo-letter & 483 & 504 & 528 & & 510 & 541 & 508 \\
Pseudo-letter/letter & 520 & 534 & 537 & & 534 & 564 & 540 \\
Pseudo-letter/pseudo-letter & 487 & 490 & 528 & & 484 & 534 & 527 \\
\hline
\end{tabular}


this analysis $(F<<1)$. For Surrounding, comparisons were faster when the second stimulus was isolated $(486 \mathrm{~ms})$ than when it was surrounded $(512 \mathrm{~ms})$. For Pair, a post hoc analysis (Bonferroni) showed that same pairs of letters received faster responses $(455 \mathrm{~ms})$ than same pairs of pseudo-letters (508 ms) and different letter/ pseudo-letter pairs $(513 \mathrm{~ms})$, and these two, in turn, were faster than the different pseudo-letter/letter pairs (539 ms).

A second ANOVA was performed in order to examine the effect of Congruence between target and surrounding. This analysis was restricted to trials in which the second stimulus of a pair was surrounded, where the surrounding shape could either be congruent or incongruent with the target. For this analysis, the within-subjects factors Pair and Congruence (congruent, incongruent) and the between-subject factor Similarity were used. There was a main effects obtained for Pair, $F(3,114)=32.23, \quad p<0.001$, but no main effects of Congruence, $F<<1$, and Similarity $(F<<1)$. A post hoc analysis (Bonferroni) of the Pair effect revealed that letter pairs were responded to fastest ( $465 \mathrm{~ms}, p<0.001$ ) followed by the pseudo-letter pairs and letter/pseudoletter pairs (both $520 \mathrm{~ms}$ ). The longest response times were obtained for pseudo-letter/letter conditions (544 ms, $p<0.05)$.

A two-way interaction was obtained between Congruence and Similarity, $F(1,38)=16.1, p<0.001$. Planned post hoc analyses showed that the Congruence effect was significant for both the Dissimilar group, $F(1$, $18)=10.5, \quad p<001$, and the Similar group, $F(1$, $20)=6.92, p<05$. Whereas for the Dissimilar group the congruence effect was positive, (congruent $=498 \mathrm{~ms}$, incongruent $=515 \mathrm{~ms}$ ), it was negative for the Similar group (congruent $=524 \mathrm{~ms}$, incongruent $=510 \mathrm{~ms}$ ).

A three-way interaction was found between Pair, Congruence, and Similarity, $F(3,114)=3.47, p<0.05$. This interaction is illustrated in Fig. 3. In order to explore this interaction, planned post hoc ANOVAs were performed separately for each experimental group.

For the Dissimilar group, a Pair (4) $\times$ Congruence (3) ANOVA revealed main effects for Pair, $F(3,54)=13.21$, $p<0.001$, and Congruence, $F(1,18)=9.73, p<0.001$, but no interaction. Letters were responded to fastest (465 ms), followed by pseudo-letters (509 ms) and mixed conditions, letter/pseudo-letter $(516 \mathrm{~ms})$ and pseudoletter/letter $(536 \mathrm{~ms})$. All conditions differed pair-wise from each other, except the letter/pseudo-letter from the pseudo-letter condition. As already observed, this group responded to congruent trials faster than incongruent ones.

For the Similar group, the same analysis revealed main effects for Pair, $F(3,60)=19.71, p<.001$, and Congruence, $F(1,20)=7, p<.01$, and a significant interaction between these factors, $F(3,60)=2.75$, $p=.045$. As in the Dissimilar condition, letters were compared fastest $(465 \mathrm{~ms})$, followed by pseudo-letters $(530 \mathrm{~ms})$ and mixed conditions, letter/pseudo-letter (524 ms) and pseudo-letter/letter $(552 \mathrm{~ms})$. Again, all conditions differed pair-wise from each other, except the letter/pseudo-letter and pseudo-letter condition. As already observed, this group responded to incongruent trials faster than to incongruent ones. The interaction between Congruence and Pair was found to be based on a large negative congruence effect for the letter/pseudoletter pairs, $F(1,20)=22.41, p<.001$; incongruent conditions were faster than congruent ones, whereas differences between congruent and incongruent stimuli did not reach significance in any of the other conditions of this group. A tendency of a negative congruence effect was recognizable for the pseudo-letter/letter condition $(p=.1)$.

\section{Discussion}

The comparison of isolated and surrounded shapes revealed that overall, letters were faster than pseudo-letters in same pairs. Given that in different pairs, fastest responses were obtained when the first one is a letter, we may infer that the categorical nature of the first stimulus is of greater importance for the speed of the response than the second. This may be explained from the fact that letters are a natural category that may be easier to represent in working memory. A fast-same effect was obtained; responses on same trials were faster than those
Fig. 3 Mean reaction times and $95 \%$ confidence intervals (vertical bars) of same pairs of letters (lele) and pseudo-letter (psps), and of different pairs of letter/pseudo-letter (leps) and pseudo-letters/letters (psle), for congruent and incongruent conditions, respectively. The results are displayed separately for the Dissimilar (left graph) and the Similar group.
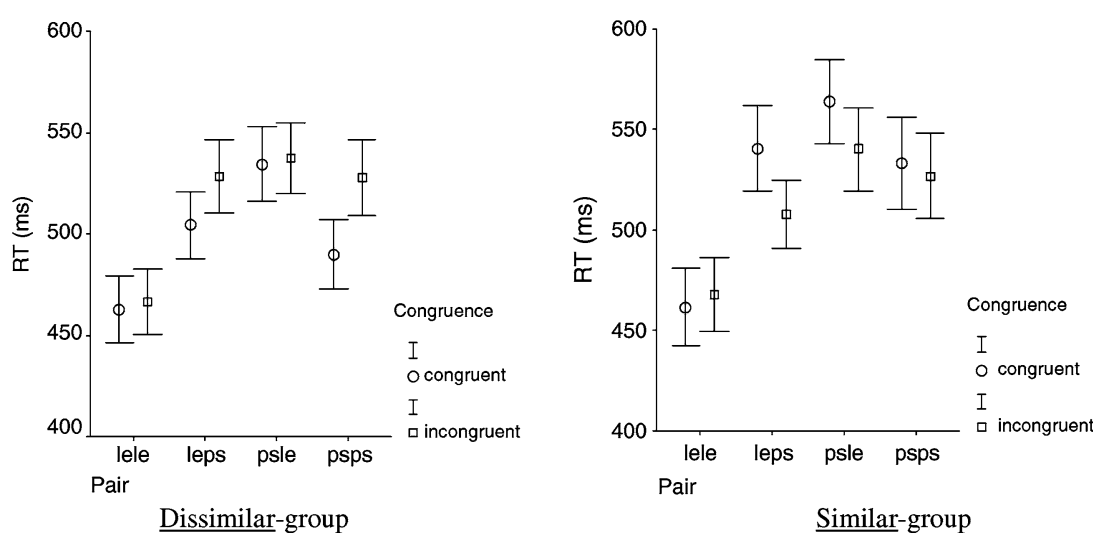
on different trials. However, in the present experiment it is unclear whether this effect is based on the physical or categorical identity, as the restricted set of stimuli did not allow us to vary same/different independently with physical and categorical identity. This was done in Experiment 2.

Same trials within the surrounded shapes yielded, as a common observation for the Dissimilar and Similar group, that there are no congruence effects for letterletter pairs. In all other respects, the results are contrasting, illustrating the impact of similarity on the task. Relatively, in the Dissimilar group, positive congruence effects and in the Similar group negative congruence effects were predominant. For pseudo-letter pairs, a congruence effect was obtained in the Dissimilar group and a null effect in the Similar group. For mixed pairs, congruence effects tended to be positive in the Dissimilar group; the positive effect occurred for pseudo-letters as second stimulus, whereas there was a null effect for letters. This result is consistent with the distinction observed for same letters and pseudo-letters in this group. For the Similar group, there were negative congruence effects in the mixed conditions. This effect is of particular interest; as van Leeuwen and Lachmann (2004) obtained negative congruence effects only for letter targets.

We expected a response conflict for the mixed pairs in the Similar condition. The presence of a response conflict was inferred from the observation that the longest response latencies occur for the different responses in the Similar condition. However, this effect was restricted in size. Therefore, we cannot be positive about whether a response conflict should be held responsible for the negative congruence effects. In order to investigate this issue, we varied the instructions between Experiments 2 and 3 .

\section{Experiment 2}

In the previous experiment, negative congruence effects were obtained when letters were followed by pseudoletters in conditions where these are similar in shape. We tentatively ascribed this result to response competition; the requirement to respond different to shapes that are similar in the context of a physical sameness task. However, a restriction is that response competition was not clearly in evidence. Another restriction was that, because of the distinction between the Similar and the Dissimilar group in Experiment 1, both groups saw only a subset of the possible pairs. As a result there were no different pairs in Experiment 1, in which either both the first and second item were letters or both were pseudoletters. This means that in order to respond correctly, it is sufficient to detect what category, letter or pseudoletter, the stimuli belong to. Experiments 2 and 3 were performed to remedy these difficulties. In both experiments, we presented the full set of possible combinations of letters and pseudo-letters. In Experiment 2, the task was the same as in the previous experiment. In contrast, in Experiment 3 the task was changed in order to check the influence of categorical judgements on the occurrence of negative congruence effects.

\section{Method}

\section{Participants}

There were 20 student volunteers ( 16 female) from the University of Leipzig between 20 and 28 years old, who were either paid or received course credits for their participation. None of these participants took part in Experiment 1. All participants had normal or corrected to normal vision and hearing.

\section{Stimuli, apparatus, and procedure}

Stimuli, apparatus, and procedure were identical to those in Experiment 1, except as described in the following. There was no distinction between a Similar and Dissimilar group in this experiment and, hence, letters and pseudo-letters of Fig. 2 were shown with equal probability in all possible combinations. Three categories of stimulus pairs were obtained: identity matches (IM), categorical matches (CM) and non-matches (NM). The IM stimulus pairs consisted of 2 (letters versus pseudo-letters) * 3 ("A", "C", 'L"), * 3 (isolated, congruent, incongruent second stimulus) $=18$ unique pairs. The CM stimulus pairs consisted of 2 (letters versus pseudo-letters) * 6 (possible combinations of " $A$ ", " $C$ ", " $\mathrm{L}$ "), * 3 (isolated, congruent, incongruent second stimulus) $=36$ unique pairs. The NM pairs consisted of 2 (letter first versus pseudo-letter first) * 6 (possible combinations of " $\mathrm{A}$ ", "C", ' $\mathrm{L}$ ") * 3 (isolated, congruent, incongruent second stimulus) $=36$ Dissimilar trials and 2 (letter first versus pseudo-letter first) * 3 ("A", "C", 'L")* 3 (isolated, congruent, incongruent second stimulus) $=18$ Similar NM trials. The 18 IM pairs are responded to as same, all the others (90 in total) as different. To equal the proportion of same and different responses, the IM pairs were repeated 5 times within a block, yielding a total of 180 trials in a block. Trials were intermixed within a block in random order; three blocks of trials were given subsequently within the experimental session, which lasted approximately $1 \mathrm{~h}$ in total.

\section{Results}

A total of 10,800 responses were collected. For removing individual outliers, the same procedure was used as in Experiment 1 . This led to the exclusion of $1.3 \%$ of the cases, which were equally distributed over the experimental conditions and individuals. Only correct responses were used for RT analyses. The overall mean RT was $525 \mathrm{~ms}(\mathrm{SD}=193 \mathrm{~ms})$ and the mean error rate was $2.4 \%$. Mean RT and error rates were not correlated. 
For this reason, analyses of variance were performed on RT data only. The data for all conditions are displayed in Table 2.

A $6 \times 2$ ANOVA including the factors Pair (identical letters versus pseudo-letters, categorical identical letters versus pseudo-letters, and mixed letter/pseudo-letter versus pseudo-letter/letter non-matching conditions) and Surrounding (isolated versus surrounded targets) revealed main effects of Pair, $F(5,95)=14.77, p<.001$, and Surrounding, $F(1,19)=25.75, p<.001$, and their interaction, $F(5,95)=4.87, p<.01$. Isolated targets $(511 \mathrm{~ms})$ showed lower RT than surrounded ones (532 ms). The IM letters were compared faster (490 ms) than IM pseudo-letters $(524 \mathrm{~ms})$. These were faster than $\mathrm{CM}$ letters and pseudo-letters $(535 \mathrm{~ms}$ and $541 \mathrm{~ms}$, respectively). The NM conditions were the slowest (letter/pseudo-letter $=542 \mathrm{~ms}$; pseudo-letter/letter $=$ $551 \mathrm{~ms})$. The interaction can be explained by the restriction of Surrounding effects to three out of the six Pair combinations: the IM letters and pseudo-letters and NM letter/pseudo-letter conditions.

In order to explore the factor Congruence, an ANOVA without isolated trials was performed, including the factors Pair and Congruence (congruent versus incongruent). This analysis revealed a main effect of Pair, $F(5,95)$ $=9.84, p<.001$, and an interaction between Pair and Congruence, $F(5,95)=2.93, p<.05$. The IM letter pairs were faster $(499 \mathrm{~ms})$ than all other pairs $(536-553 \mathrm{~ms})$, among which there was no difference. The interaction was found to be due to a positive congruence effect for IM pseudo-letter pairs contrasting to a negative congruence effect in NM pseudo-letter/letter pairs and missing congruence effects in all other cases.

The effect of Similarity on Congruence was explored by a specific follow-up analysis of NM pairs. These are mixed, consisting of a letter and a pseudo-letter and correspond to the pairs for which negative congruence effects were found depending on similarity in the previous experiment. The analysis used the factors Pair (letter/pseudo-letter versus pseudo-letter/letter), Similarity (similar in form, e.g. a combination of similar items,

Table 2 Mean reaction times (ms) for isolated, congruent and incongruent items, for IM and CM and for similar and dissimilar NM conditions of Experiment 2

\begin{tabular}{llll}
\hline & Isolated & Congruent & Incongruent \\
\hline IM & & & \\
Letter/letter & 471 & 498 & 501 \\
Pseudo-letter/pseudo-letter & 499 & 528 & 545 \\
CM & & & \\
Letter/letter & 531 & 529 & 544 \\
Pseudo-letter/pseudo-letter & 545 & 533 & 546 \\
NM & & & \\
Letter/pseudo-letter & 553 & 571 & 553 \\
$\quad$ Similar & 522 & 532 & 549 \\
$\quad$ Dissimilar & 577 & 604 & 557 \\
Pseudo-letter/letter & 529 & 542 & 540 \\
$\quad$ Similar & & & \\
Dissimilar & & \\
\hline
\end{tabular}

letter " $A$ " and pseudo-letter " $A$ ", versus a combination of dissimilar items, letter " $A$ " and pseudo-letter " $C$ "), and Congruence (congruent versus incongruent). The results are shown in Fig. 4. The only significant main effect was found for Similarity, $F(1,19)=22.22, p$ $<.001$. Interactions were found between Pair and Congruence, $F(1,19)=4.92, p<.05$, and between Similarity and Congruence, $F(1,19)=12.14, p<.001$. The first interaction is due to a negative congruence effect for the pseudo-letter/letter condition that was absent in the other Pair conditions. The second interaction is due to negative congruence effects for similar items that were absent (with a small positive tendency) for dissimilar ones.

\section{Discussion}

We observed a preference for isolated targets as well as the fast-same effect for letters and shapes. This effect is larger for letters than for pseudo-letters. Furthermore, we obtained positive congruence effects for most conditions, except for the letter IM condition and the pseudo-letter/letter NM condition. In the first case, no congruence effect was observed. That letters did not yield a congruence effect in IM conditions, whereas pseudo-letters did, is comparable to the Dissimilar group of Experiment 1. It is appropriate to compare these results to those of the Dissimilar group of Experiment 1 , because in the present experiment, similar trials are only a fraction of the total.

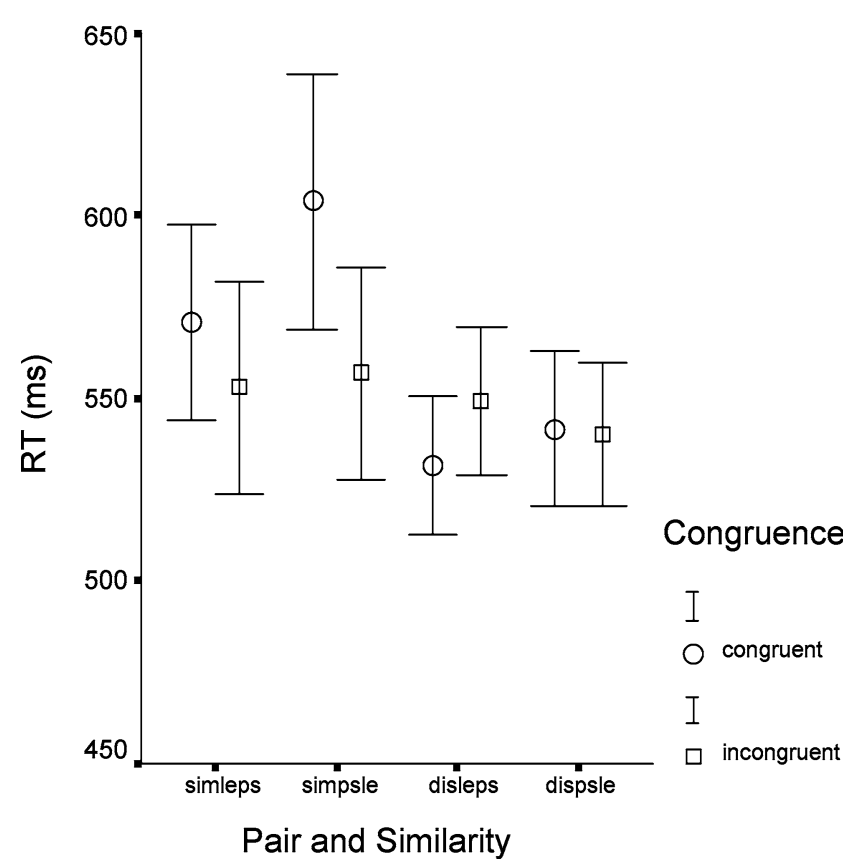

Fig. 4 Mean reaction times and 95\% confidence intervals (vertical bars) of NM letter/pseudo-letter and pseudo-letter/letter pairs for similar and dissimilar conditions and for congruent and incongruent surroundings 
When we focus our attention on the similar trials, it turns out that these show strong negative congruence effects, comparable to those of the Similar group of Experiment 1. However, compared to Experiment 1, these trials showed evidence of a response conflict more clearly; they are much slower, on average, than the dissimilar trials. We may, therefore, conclude that in the present task negative congruence effects were co-extensive with response conflict.

\section{Experiment 3}

The results of Experiment 1 did not allow us to distinguish between effects that were based on physical and categorical identity. In the terminology of the previous experiment, this is because of the absence of CM trials in Experiment 1. In Experiment 2, we have seen that CM trials invariably produce congruence tendencies. Their presence in the experiment seems to be irrelevant with respect to the occurrence of the negative congruence effects. This observation contrasts with that of van Leeuwen and Lachmann (2004), where the categorical nature of stimuli played an important role in the occurrence of positive or negative congruence effects. The present experiment focused the task on the categorical nature of the stimuli (letters versus shapes). A categorical same-different task was used, where IM as well as CM pairs were responded to as same.

In the categorical sameness task of Lachmann and van Leeuwen (2004b) and Lachmann and Geissler (2002), Similarity between the stimuli did not have a crucial role as catalyst of response competition, unlike in the physical sameness task (Lachmann 2001; Lachmann and van Leeuwen 2004a, c). Physical similarity is irrelevant for categorical sameness, and for this reason it is less likely to influence the task. In accordance with this assumption, we expected for the present experiment, unlike the previous one, no effect of Similarity.

\section{Method}

\section{Participants}

There were 21 student volunteers ( 15 female) from the University of Leipzig between 20 and 29 years old, who were either paid or received course credits for their participation. None of these participants took part in any other experiment of the present study. All participants had normal or corrected to normal vision and hearing.

\section{Stimuli, apparatus, and procedure}

Stimuli, apparatus, and procedure were identical to Experiment 2, except that participants responded to IM and CM pairs with same and with different only to NM pairs. As a consequence, no repetitions of IM, CM, or
NM had to be included in order to balance same and different responses. Thus, a block consisted of 108 trials and a session consisted of five blocks, resulting in the same number of total trials as in Experiment 1. A session lasted approximately $1 \mathrm{~h}$.

\section{Results}

A total of 11,340 responses were collected. For removing individual outliers, the same procedure was used as in Experiment 1. This led to the exclusion of $1.7 \%$ of the cases, which were equally distributed over the experimental conditions and individuals. Only correct responses were used for RT analyses. The overall mean $\mathrm{RT}$ was $680 \mathrm{~ms}(\mathrm{SD}=251 \mathrm{~ms})$ and the mean error rate was $4.6 \%$. Mean RT and error rates were not correlated. For this reason, analyses of variance were performed on RT data only. The data for all conditions are displayed in 'Table 3.

The same set of analyses as for Experiment 2 was run. The Pair (6) $\times$ Surrounding (2) ANOVA resulted in significance for Pair, $F(5,100)=36.6, p<.001$, as well as for Surrounding, $F(1,20)=17.49, p<.001$. No interaction was found. Pairs were compared $20 \mathrm{~ms}$ faster when the second item was isolated than when it was surrounded $(687 \mathrm{~ms})$. The IM letter pairs were fastest (475 ms) followed by CM letters $(620 \mathrm{~ms})$ followed by NM letter/pseudo-letter (678 ms) and IM pseudo-letters (685 ms), which did not differ from each other. The slowest responses were obtained for NM pseudo-letter/ letter pairs $(728 \mathrm{~ms})$ and CM pseudo-letters $(732 \mathrm{~ms})$, which did not differ from each other.

The Pair (6) $\times$ Congruence (2) ANOVA resulted in main effects of Pair, $F(5,100)=33.16, p<.001$, and Congruence, $F(5,95)=2.93, p<.05$. The effects of Pair resemble those of the previous ANOVA; congruent conditions received faster responses $(680 \mathrm{~ms})$ than incongruent ones $(694 \mathrm{~ms})$.

The Pair $(2) \times$ Similarity $(2) \times$ Congruence $\quad(2)$ ANOVA on NM RTs revealed a main effect of Pair, $F(1$,

Table 3 Mean reaction times (ms) for isolated, congruent and incongruent items, for IM and CM and for similar and dissimilar NM conditions of Experiment 3

\begin{tabular}{llll}
\hline & Isolated & Congruent & Incongruent \\
\hline IM & & & \\
Letter/letter & 554 & 584 & 586 \\
Pseudo-letter/pseudo-letter & 665 & 677 & 714 \\
CM & & & \\
Letter/letter & 615 & 617 & 629 \\
$\quad$ Pseudo-letter/pseudo-letter & 719 & 734 & 743 \\
NM & & & \\
Letter/pseudo-letter & 660 & 675 & 697 \\
$\quad$ Similar & 673 & 668 & 695 \\
$\quad$ Dissimilar & & & \\
Pseudo-letter/letter & 711 & 724 & 751 \\
$\quad$ Similar & 710 & 740 & 734 \\
$\quad$ Dissimilar &
\end{tabular}


$20)=15.44, p<.001$, responses were faster when the first item was a letter followed by a pseudo-letter $(683 \mathrm{~ms})$ than when it was a pseudo-letter followed by a letter $(737 \mathrm{~ms})$, and a main effect of Congruence, $F(1$, $20)=5.56, p<.05$, congruent condition were advanced (702 ms) as compared with incongruent ones $(717 \mathrm{~ms})$. No other main effects or interactions were evident.

\section{Discussion}

The analysis of isolated versus surrounded conditions showed an overall preference for isolated stimuli and a fast-same effect that was restricted to IM letter stimuli. Overall, there was a large difference between letter and pseudo-letter conditions, which was not observed for the physical sameness task of Experiments 1 and 2. Given that in the mixed conditions (NM), response times were slowest when the first item is a pseudo-letter, we may ascribe the difficulty to the representation of pseudo-letters in working memory, compared to that of letters. We may speculate that the latter have a compact phonological representation that is lacking in pseudo-letter shapes.

Congruence effects prevailed in this experiment more consistently than in the previous ones. They were absent for IM letter pairs, however, as in the previous experiments. No negative congruence effects were obtained. This was expected on the assumption that there are no response conflicts due to Similarity in this task. Correspondingly, there were no differences between the Similar and Dissimilar NM trials. In addition, the NM trials were no longer the ones with the slowest responses. In this experiment, response conflicts do not seem to be a determinant of response time. The absence of both negative congruence effects and response conflicts constitutes additional evidence for their co-extensive character in the same-different task.

\section{General discussion}

In three experiments, the role of Congruence of a surrounding frame of the second stimulus in a same-different task was investigated, in which letters as well as pseudo-letters were presented. In the first two experiments, a physical sameness task was used, in which participants respond with same when both targets are identical in shape but different otherwise. Congruence effects were observed for letters and pseudo-letters. They were more consistently obtained for pseudo-letters than for letters. This distinction replicates in a weaker form the dissociation obtained for letters and pseudo-letters in the choice-response tasks of van Leeuwen and Lachmann (2004), where letters showed a negative congruence effect. That the distinction was weaker suggests that the categorical distinction of letters versus pseudo-letters was not so important for the present task.

In Experiment 1, we observed negative congruence effects for conditions in which the non-matching stimuli were similar. The effect occurred both when a letter followed a pseudo-letter and when a pseudo-letter followed a letter. We proposed, tentatively, to ascribe this result to response competition. When the physical shapes were similar but the task was to respond different, a congruent surrounding shape that emphasizes the similarity interfered with the correct response. The extra time in these conditions was the time it took to resolve this competition. The source of the effect, therefore, is different from that in van Leeuwen and Lachmann (2004), where the categorical nature of the stimuli was involved in the occurrence of negative congruence effects by determining the perceptual strategy, with which the stimulus was encoded.

The possibility that the categorical nature of the stimuli was involved could not be excluded from the results of Experiment 1 alone. The reason was that, as a methodological consequence of the choice to present Similar and Dissimilar stimuli to different groups of participants, the variation in the stimuli was restricted such that, in principle, they could perform the task as a categorical task: the absence of letter pairs and pseudoletter pairs in different trials implies that the same response could be made using the criterion whether or not both stimuli on a trial were from the same category. In addition, the response times of the trials in which we predicted a response conflict were only a little bit longer than the ones for which it was not predicated, so the evidence for an actual response conflict in this experiment is relatively weak.

To compensate for the restrictions of Experiment 1, we performed Experiments 2 and 3. These experiments use all possible combinations of stimuli, avoiding the restrictions of Experiment 1. As a result, they had the inevitable drawback that the proportion of trials for which a response conflict is expected is relatively low. Experiment 2 confirmed the observation that the central characteristic of the physical sameness task was the occurrence of negative congruence effects in the different condition, when the first and the second stimulus were similar, but not identical. As the response times where this effect occured were largest overall, this effect could now be ascribed to response competition unambiguously.

That negative congruence effects are co-extensive with the response competition was confirmed in Experiment 3, where a categorical sameness criterion was used. Participants responded with same when the two targets were either both letters or both pseudo-letters, and different if one was a letter and the other a pseudoletter. The task to detect categorical rather than physical identity reduced the role of similarity. Thus, it removed the basis for a response conflict in the conditions in which letters and pseudo-letter pairs were presented. As a result, the conditions in which these pairs were similar in their physical shape were no longer the ones with the longest response times. In these conditions also, the negative congruence effects for these stimuli disappeared. We may conclude that response conflicts 
involving similarity and negative congruence effects arrive and leave together.

A further distinctive characteristic of the categorical sameness task (Experiment 3) is that there was no fastsame effect for pseudo-letters, suggesting that pseudoletter identity was more difficult to process than letter identity. We raised the question whether this interesting effect is based on a memory or perceptual representation. From the observation that in all categorical conditions, RTs are invariably high when the first stimulus is a pseudo-letter, we may conclude that the workingmemory representation of pseudo-letters had the highest contribution to the difficulty of the task. A similar observation was made for Experiment 1. We may speculate that this is because letters form a natural category, and are represented in a more efficient way, for instance, a phonemic code (for a controlled study on the role of category representation efficiency in the same-difference task, we refer to Lachmann and van Leeuwen 2004a, b, c). These results indicate that the categorical effects now predominantly play a role in the memory representation, rather than in the perceptual one, as in the corresponding choice-response task (van Leeuwen and Lachmann 2004). An interesting possibility might have been that the categorical nature of the stimuli in working memory operates to influence the perceptual representation of the second stimuli-and through this on the congruence effects. However, we did not observe any systematic effects of this kind.

The upshot of the present results is that we have identified in response conflicts a novel source of negative congruence effects. This source is independent of the feature integration strategy observed in van Leeuwen and Lachmann (2004) and both seem to be, to some extent, mutually exclusive.

Acknowledgements The authors would like to thank anonymous reviewers for helpful suggestions.

\section{References}

Bavelier D, Deruelle C, Proksch J (2000) Positive and negative compatibility effects. Percept Psychophys 62:100-112

Bjork EL, Murray JT (1977) On the nature of input channels in visual processing. Psychol Rev 84:472-484

Bouma H (1970) Interaction effects in parafoveal letter recognition. Nature 226:177-178

Briand KA (1994) Selective attention to global and local structure of objects: alternative measures of nontarget processing. Percept Psychophys 55:562-574

Chung ST, Levi DM, Legge GE (2001) Spatial-frequency and contrast properties of crowding. Vision Res 41:1833-1850

Egeth HE, Santee JL (1981) Conceptual and perceptual components in interletter inhibition. J Exp Psychol 7:506-517

Eriksen BA, Eriksen CW (1974) Effects of noise letters upon the identification of a target letter in a nonsearch task. Percept Psychophys 16:143-149
Estes WK (1972) Interactions of signal and background variables in visual processing. Percept Psychophys 12:278-286

Estes WK (1982) Similarity-related channel interactions in visual processing. J Exp Psychol 8:353-382

Freeman E, Driver J, Sagi D, Zhaoping L (2003) Top-down modulation of lateral interactions in early vision: does attention affect integration of the whole or just perception of the parts? Curr Biol 13:985-989

Hagenaar R, van der Heijden AH (1986) Target-noise separation in visual selective attention. Acta Psychol 62:161-176

Keren G, O'Hara WP, Skelton JM (1977) Levels of noise processing and attentional control. J Exp Psychol 3:653-664

Krueger LE (1973) Effect of irrelevant surrounding material on speed of same-different judgement of two adjacent letters. J Exp Psychol 98:252-259

Lachmann T (2001) Strategies of coding and processing in a physical same-different task. In Sommerfeld E, Kompass R, Lachmann T (eds) Proceedings of the International Society for Psychophysics. Pabst, Lengerich, pp 98-104

Lachmann T, Geissler H-G (2002) Memory search instead of template matching? Representation-guided inference in samedifferent performance. Acta Psychol 111:19-43

Lachmann T, van Leeuwen C (2004a) Memory-guided inference in same-different comparison tasks. In: Kaernbach C, Schroeger E, Müller HJ (eds) Psychophysics beyond sensation. Scientific Psychology Series. Erlbaum, Hilldale, NJ, pp 199-221

Lachmann T, van Leeuwen C (2004b) Individual pattern representations are context-independent but their collective representation is context-dependent. Q J Exp Psychol A (in press)

Lachmann T, van Leeuwen C (2004c) Task-invariant aspects of Goodness in perceptional representations. Q J Exp Psychol A (in press)

Lavie N (1995) Perceptual Load as a necessary condition for selective attention. J Exp Psychol 21:451-468

Leat SJ, Li W, Epp K (1999) Crowding in central and eccentric vision: the effects of contour interaction and attention. Invest Ophthalmol Visual Sci 40:504-512

van Leeuwen C, Bakker L (1995) Stroop can occur without Garner interference: strategic and mandatory influences in multidimensional stimuli. Percept Psychophys 57:379-392

van Leeuwen C, Lachmann T (2004) Negative and positive congruence effects in letters and shapes. Percept Psychophys (in press)

Miller J (1991) The flanker compatibility effect as a function of visual angle, attentional focus, visual transients, and perceptual load: a search for boundary conditions. Percept Psychophys 49:270-88

Nazir TA, Jacobs AM, O'Regan JK (1998) Letter legibility and visual word recognition. Mem Cognit 26:810-821

Nickerson RS (1969) "Same-different"- response times: A model and a preliminary test. Acta Psychol 30:257-275

Pomerantz JR, Pristach EA, Carson CE (1988) Attention and object perception. In: Shepp B, Ballesteros S (eds) Object perception: structure and process. Erlbaum, Hillsdale, NJ, pp 5389

Posner MI (1978) Chronometric explorations of mind. Erlbaum, Hillsdale, NJ

Proctor RW (1981) A unified theory for matching task phenomena. Psychol Rev 88:291-326

Rueckl JG, Suzuki S, Yeh SL (1991) On the locus of redundancy effects in a letter-detection task. Percept Psychophys 49:412-421

Sanders AF, Lamers JM (2002) The Eriksen flanker effect revisited. Acta Psychol 10:41-56

Toet A, Levi DM (1992) Spatial interaction zones in the parafovea. Vision Res 32:1349-1357

Treisman AM, Gelade G (1980) A feature integration theory of attention. Cognit Psychol 12:97-136 